\title{
ZnO NANOPARTICLES: SYNTHESIS AND EVOLUTION
}

\author{
1,2Vojtěch PŘIKRYL, 1,2Zuzana BYTEŠNíKOVÁ, 1,2Pavel ŠVEC, 1,2Lukáš RICHTERA, \\ 1,2 Vojtěch ADAM
}

${ }^{1}$ Department of Chemistry and Biochemistry, Mendel University in Brno, Brno, Czech Republic, EU

${ }^{2}$ Central European Institute of Technology, Brno University of Technology, Brno, Czech Republic, EU

https://doi.org/10.37904/nanocon.2020.3771

\begin{abstract}
$\mathrm{ZnO}$ is a material that is biocompatible, biodegradable, and has low toxicity. Recently it has emerged as an interesting material for use in biomedicine, cosmetics and agriculture. Chemical synthesis of $\mathrm{ZnO}$ nanoparticles was investigated due to its potential ease of preparation and upscalability. Ethanol was used as a solvent that promotes the continued growth of the as-prepared nanoparticles. This work presents facile methods of $\mathrm{ZnO}$ nanoparticle preparation and a potential method for $\mathrm{ZnO}$ nanowire growth at room temperature.
\end{abstract}

Keywords: Zinc oxide, nanoparticles, nanowires, chemical synthesis

\section{INTRODUCTION}

$\mathrm{ZnO}$ is a material with promising potential for use in various fields including biomedicine, cosmetics and agriculture [1-4] due to its biocompatibility, biodegradability, and low toxicity [5]. Nanoparticles are used in the same fields for their high surface to volume ratio, and antibacterial properties [6,7]. Studies show that nanoparticles exhibit different properties depending on their shape and size [8,9]. As such new methods for synthesis of nanoparticles of various dimensions are needed. Chemical synthesis offers a way to synthesize nanoparticles in an inexpensive and easy way without the use of specialized equipment $[10,11]$. In this case, it also allows for use of environmentally friendly materials which is crucial in the above mentioned fields. Due to the increased use of nanoparticles in highly industrialized fields such as agriculture, there is a need for methods that allow for the synthesis of large volumes of nanoparticles. At such scales storage and transportation form a massive obstacle due to their prohibitive expensiveness. One method that allows to reduce the volume of nanoparticles is lyophilization. However, lyophilization can alter the parameters of nanoparticles [12] and so a method that synthesizes nanoparticles that are stable even after lyophilization is needed. This study proposes multiple methods for synthesis of $\mathrm{ZnO}$ nanoplates, spherical nanoparticles, and nanowires of various shapes and dimensions that are inexpensive, simple, upscalable, and stable after lyophilization.

\section{MATERIALS AND METHODS}

\subsection{Materials and equipment}

All chemicals in this study, unless otherwise stated, were purchased from Sigma-Aldrich (St. Louis, MO, USA). The demineralized water was produced using reverse osmosis apparatus Aqual 25 (Aqua Osmotic, Tišnov, Czech Republic). The demineralized water was further treated by the Milli-Q Direct QUV equipment furnished with a UV lamp (Aqua Osmotic, Tisnov, Czech Republic). The resistance value was $18.20 \mathrm{M} \Omega \cdot \mathrm{cm}\left(\right.$ at $25^{\circ} \mathrm{C}$ ). The drying oven used for drying (if applicable) was Memmert UM 400 (Memmert, Schwabach, Germany). The oven used for calcination was $L$ laboratory chamber furnace (LAC, Rajhrad, Czech Republic). Each calcination process included a $30 \mathrm{~min}$ ramping time prior to calcination at $400{ }^{\circ} \mathrm{C}$ for $3 \mathrm{~h}$ and cooling to room temperature 
overnight. Lyophilization was performed by FreeZone 2.5 Liter Benchtop Freeze Dryer at $0.014 \mathrm{mBar},-55^{\circ} \mathrm{C}$ overnight (Labconco, Kansas City, MO, USA).

\subsection{Preparation of $\mathrm{ZnO}$ nanoplates}

$\mathrm{ZnO}$ nanoplates preparation was based on a method reported elsewhere [13]. The method consists of dropwise addition of $33.5 \mathrm{ml}, 0.1 \mathrm{M} \mathrm{NaOH}$ (Mikrochem, Olomouc, Czech Republic) into $30 \mathrm{ml}$ of $0.1 \mathrm{M}$ $\mathrm{ZnSO}_{4} \cdot 7 \mathrm{H}_{2} \mathrm{O}$. The resulting mixture was stirred at $820 \mathrm{rpm}$ for $15 \mathrm{~min}$ at room temperature and then washed several times with Milli-Q water. A scaled up version of the preparation was performed by using $10 x$ the amount of the above-mentioned chemicals with unchanged concentrations. The samples were lyophilized to test their resistance to damage caused by lyophilization.

\subsection{Preparation of spherical $\mathrm{ZnO}$ nanoparticles}

Spherical $\mathrm{ZnO}$ nanoparticles were prepared via method reported by Chung et al. [14]. A sol-gel was formed by dropwise addition of $0.1 \mathrm{M}$ oxalic acid dihydrate (Lach-Ner, Neratovice, Czech Republic) in $60 \mathrm{ml} 99.8 \%$ ethanol (Lach-Ner) into $0.1 \mathrm{M}$ zinc acetate dihydrate in $40 \mathrm{ml}$ of $99,8 \%$ ethanol (Lach-Ner) heated to $60{ }^{\circ} \mathrm{C}$. The resulting mixture was stirred at $820 \mathrm{rpm}$ for $90 \mathrm{~min}$ and then dried overnight at $60^{\circ} \mathrm{C}$. The dried powder was calcinated and redispersed in $50 \mathrm{ml}$ of Milli-Q water. A scaled up synthesis was performed by using $10 \mathrm{x}$ the amount of the used chemicals with unchanged concentrations. Experiment using the original procedure with double concentration of oxalic acid was also performed.

\subsection{Preparation of $\mathrm{ZnO}$ nanowires}

$\mathrm{ZnO}$ nanowires were prepared by modification of the method outlined in 2.3. The nanowire seed solution was made from $0.1 \mathrm{M}$ oxalic acid dihydrate (Lach-Ner) in $60 \mathrm{ml} 99.8 \%$ ethanol (Lach-Ner) which was added dropwise into $0.1 \mathrm{M}$ zinc acetate dihydrate in $40 \mathrm{ml}$ of $99.8 \%$ ethanol (Lach-Ner) heated to $60{ }^{\circ} \mathrm{C}$ and stirred at $500 \mathrm{rpm}$. The resulting mixture was left to age. After 1 month of aging at room temperature the solution was topped to $100 \mathrm{ml}$ with $99.8 \%$ ethanol to compensate for ethanol evaporation. Then the solution was washed several times with Milli-Q water. This procedure was scaled up using $10 \times$ the amount of the necessary chemicals with unchanged concentrations. The scaled up product was split into three aliquots. The first aliquot was simply washed with Milli-Q water. The second was washed with Milli-Q water, redispersed in water, dried at $60^{\circ} \mathrm{C}$ overnight and calcinated. The third aliquot was dried overnight at $60^{\circ} \mathrm{C}$ without washing and calcinated.

\subsection{Scanning electron microscopy}

The samples were examined by SEM on a Tescan MAIA 3 equipped with a FEG (Tescan Ltd., Brno, Czech Republic). The best pictures were obtained using the In-lens SE detector at working distance between $2.91-3.06 \mathrm{~mm}$ and at $5 \mathrm{kV}$ acceleration voltage. $768 \times 858$ pixel images were obtained at $1,500-100,000$ fold magnification covering sample area of $2.08-138.00 \mu \mathrm{m}$. Full frame capture was performed in UH resolution mode and accumulation of image with image shift correction was enabled, and it took about 0.5 minutes with the $\sim 0.32 \mu \mathrm{s} /$ pixel dwell time. Spot size was set at $2.4 \mathrm{~nm}$.

\subsection{Image analysis}

The nanoparticle parameters stated in this study were determined via image analysis using the ImageJ software (ver. 1.53c). The dimensions of $\mathrm{ZnO}$ nanoplates were determined by fitting an ellipse to the hexagonal nanoparticles in order to get an estimate of their length and width from the major and minor ellipse axes. Spherical nanoparticle diameters were determined using the Analyze Particles function with a suitable threshold. The length and width of $\mathrm{ZnO}$ nanowires were determined by fitting a rectangle to the nanowire outline. 


\section{RESULTS}

\subsection{ZnO nanoplates}

SEM micrograph of $\mathrm{ZnO}$ nanoplates by the method outlined in 2.1. is shown in Figure 1.

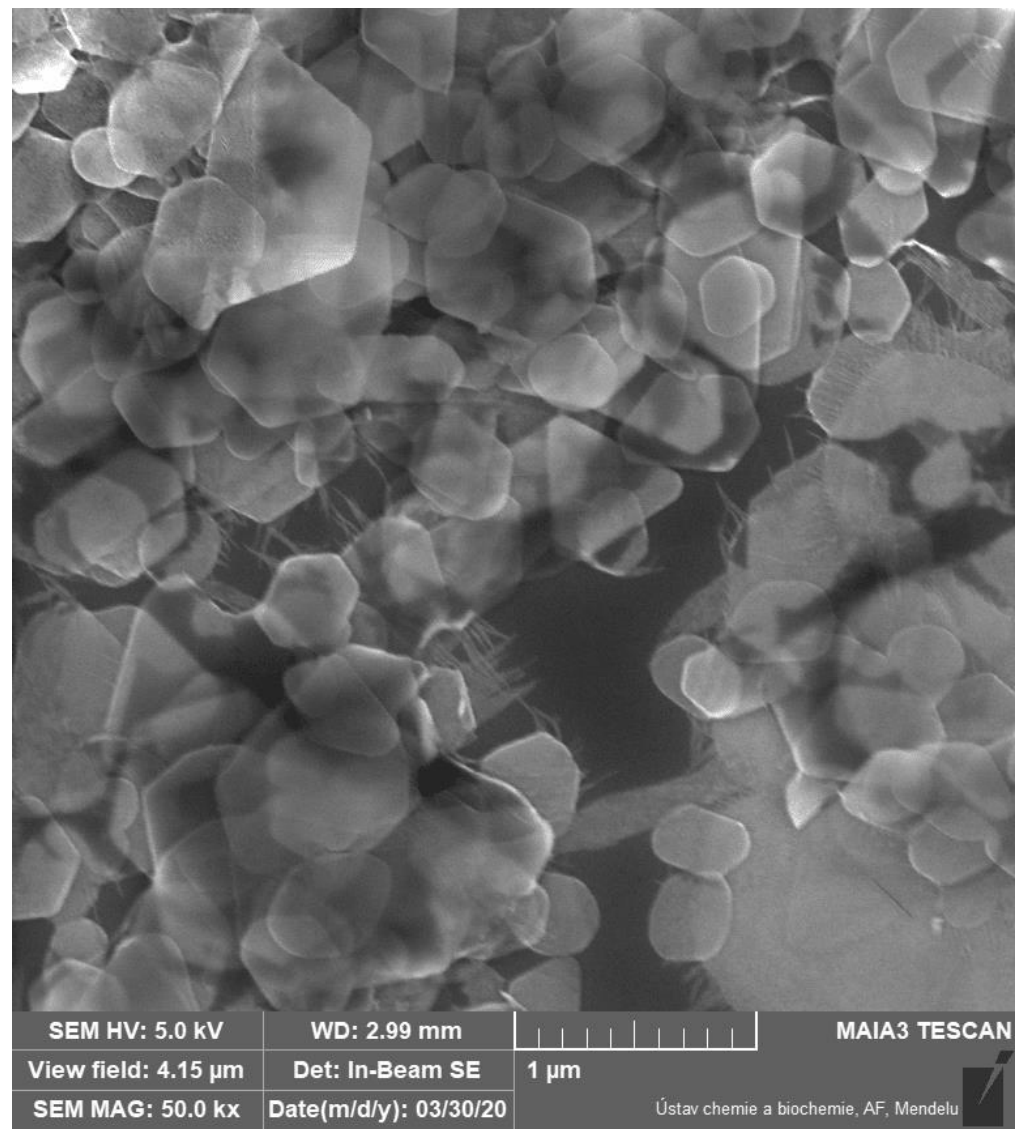

Figure 1 SEM micrograph of $\mathrm{ZnO}$ nanoplates

From the Figure 1 we can see that nanoplates with a wide range of sizes were synthesized, with the smallest having a length of around $0.5 \mu \mathrm{m}$ and the largest having over $3 \mu \mathrm{m}$. The structure of the nanoplates is mostly of hexagonal crystals which is in agreement with theoretical structure of $\mathrm{ZnO}$ which is hexagonal wurtzite [15, 16]. This synthesis proved to be easily upscalable by simple multiplication of the amount of used chemicals and shown only small structural modification when lyophilized as evidenced by Figure 2. From the figure it is clear that while the structure in Figure 2a) of the nanoplates is nearly identical to that of Figure 1 the structure of Figure $\mathbf{2 b}$ ) shows nanoplates with visibly larger length and width but otherwise seemingly unchanged. The simple preparation, ease of upscalability and relatively small structural modification of the sample by lyophilization suggest the synthesis is interesting in regards to industrialization and mass production.

\subsection{Spherical $\mathrm{ZnO}$ nanoparticles and $\mathrm{ZnO}$ nanowires}

Spherical ZnO nanoparticles were synthesized as described in section 2.3. SEM micrograph of these particles is shown in Figure 3a). The figure shows that spherical nanoparticles with diameter ranging from 30 to $80 \mathrm{~nm}$ were formed with the average nanoparticle size being around $50 \mathrm{~nm}$. Further experiments with increasing concentration of oxalic acid were made. When the concentration of used oxalic acid was doubled an interesting phenomena occurred. As shown in Figure $\mathbf{3 b}$ ) the nanoparticles had a tendency to aggregate and in some cases they formed chainlike structures. 

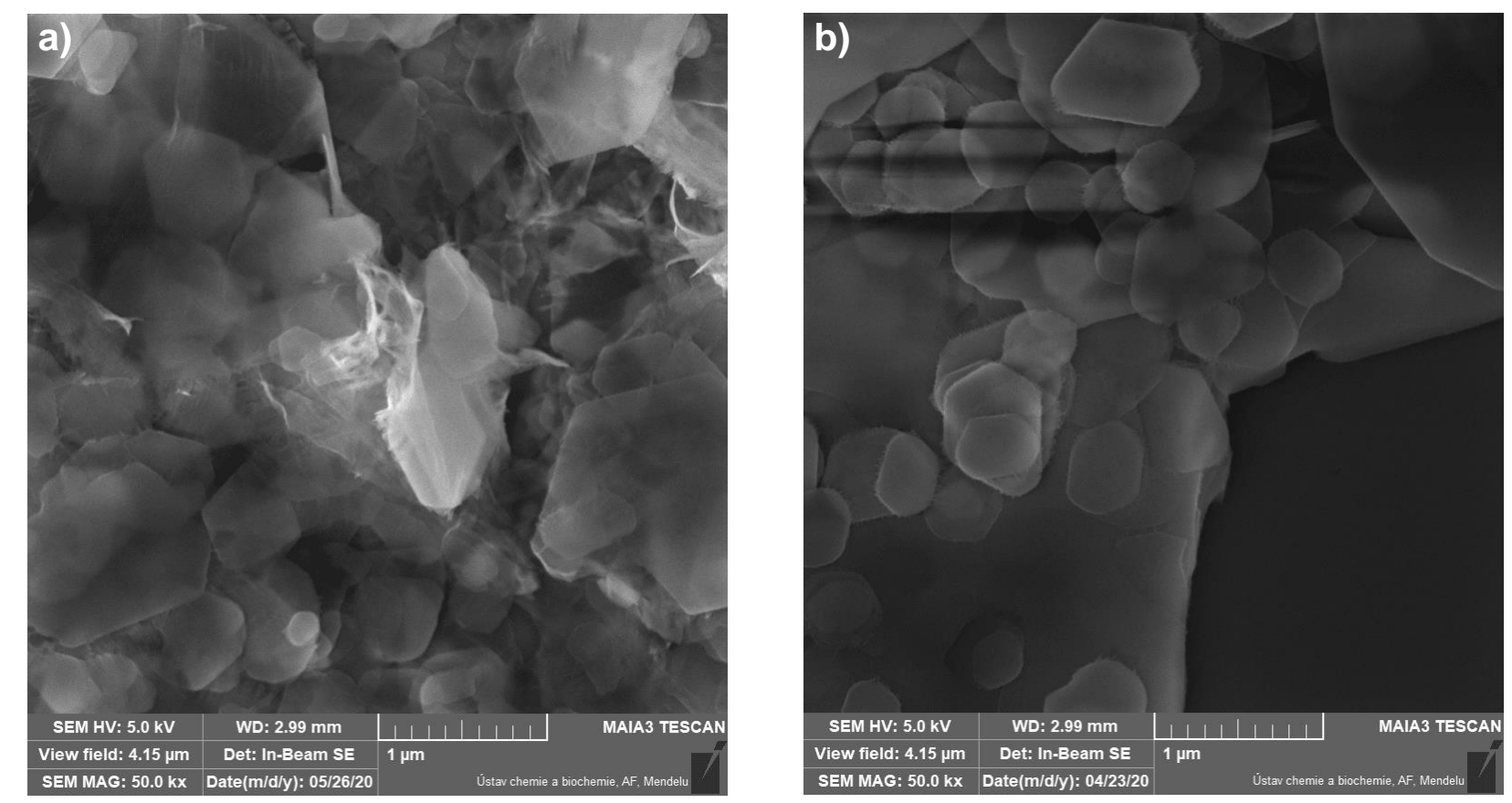

Figure 2 SEM micrograph of nanoplates: a) 10x upscaled synthesis, b) ZnO nanoplates after lyophilization
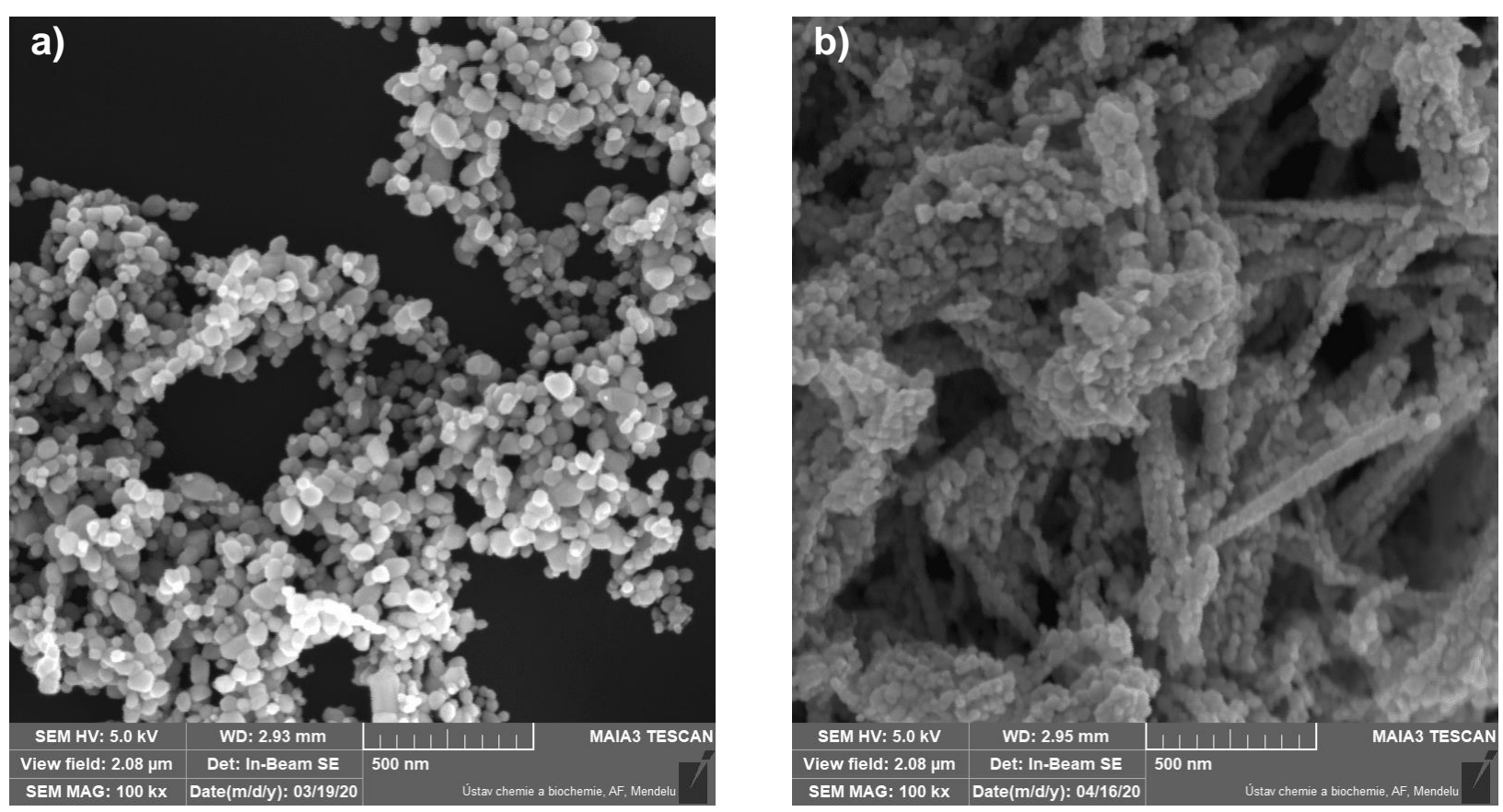

Figure 3 SEM micrograph: a) spherical ZnO nanoparticles, b) nanoparticles made with $2 \times$ concentration of oxalic acid

This result suggested that by modification of the procedure 1D nanostructures could be created. Consequently, a modified synthesis was performed as described in section 2.4. Ethanol was used as aging dispersant because it was previously shown to facilitate 1D nanostructure growth at room temperature [17]. Figure 4 shows the SEM micrographs of the $\mathrm{ZnO}$ nanowires created using this method. The figure shows nanowires with length of over $10 \mu \mathrm{m}$ with some up to $50 \mu \mathrm{m}$ long. The thickness of the wires ranges between $0.2 \mu \mathrm{m}$ to $0.6 \mu \mathrm{m}$. The wires are not uniform in thickness with the central parts being noticeably wider than the ends which are narrowed into sharp tips. Further studies are necessary to determine the dimensions of the wires in 


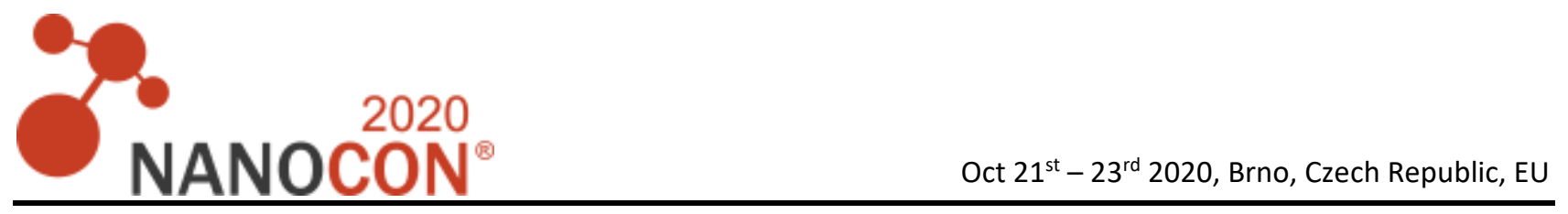

different stages of the growth process. Figure 5 shows the comparison of the three aliquots made from the upscaled preparation of $\mathrm{ZnO}$ nanowires (2.4.).
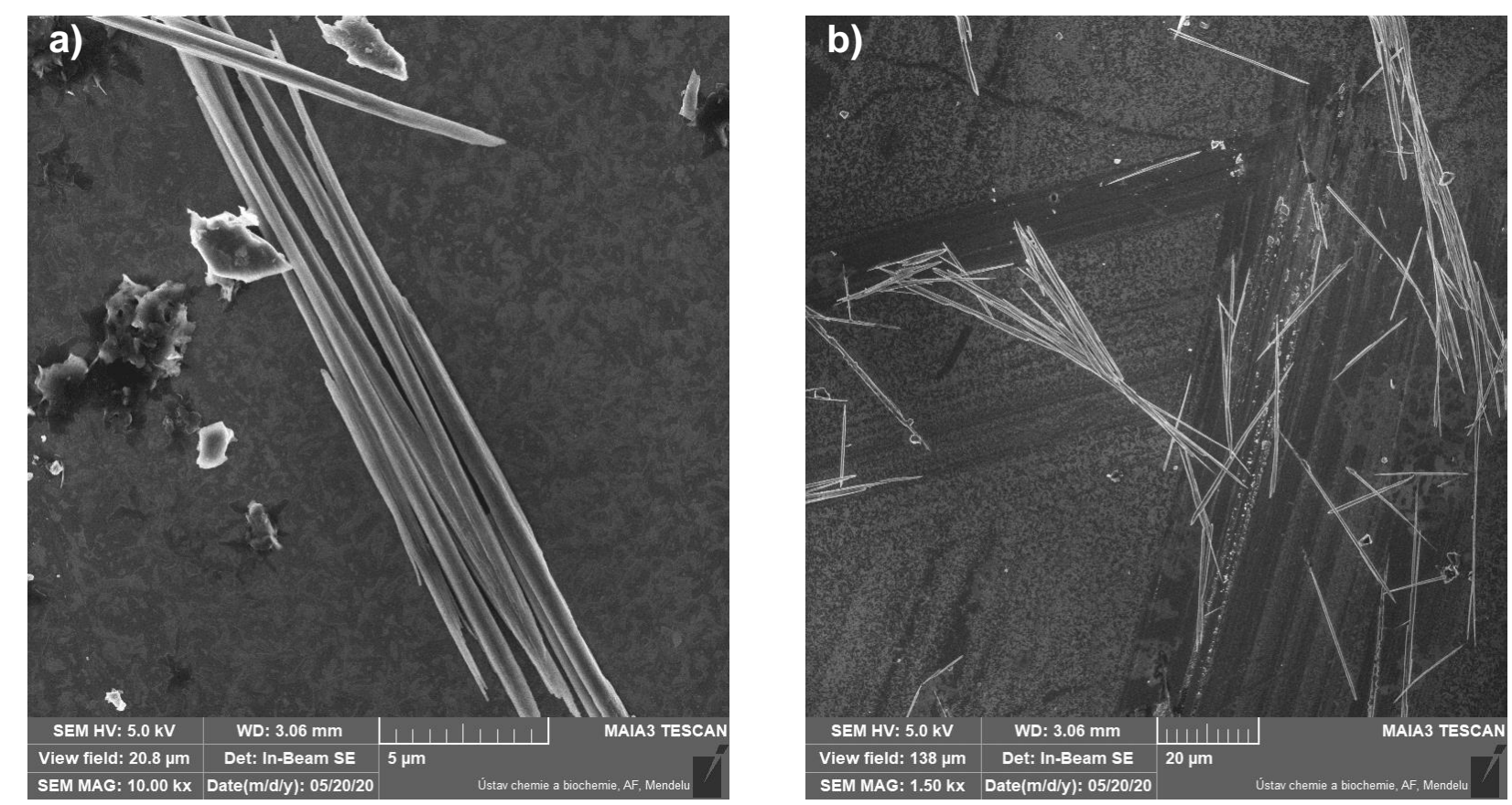

Figure 4 SEM micrograph of ZnO nanowires: a) close-up, b) wide view
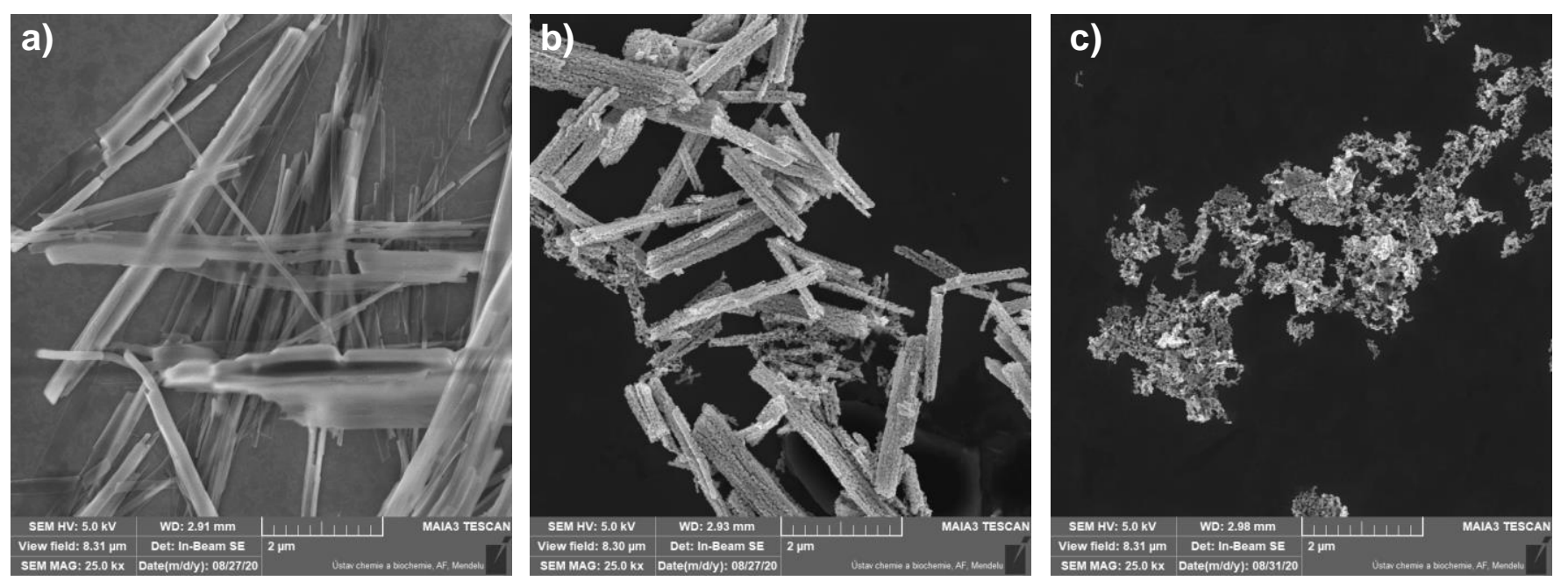

Figure 5 SEM micrographs of upscaled solution: a) only washed, b) washed and calcinated, c) only calcinated

Figure 5a) shows nanowires that are similar to the original synthesis result. Figure $5 b$ ) shows a structure that is similar to nanowires composed from the smaller spherical nanoparticles. Figure 5c) shows that simple calcination caused the structure to revert to the spherical nanoparticles that were formed with no aging in ethanol involved.

\section{CONCLUSION}

This study shows multiple methods for facile and inexpensive synthesis of $\mathrm{ZnO}$ nanoplates and nanowires. The nanoplates can be prepared quickly, are simple to upscale and can be lyophilized with only little alteration 
of their structure. This study also shows a method for evolution of spherical nanoparticles into nanowires using only ethanol at room temperature. Both methods show promise regarding mass production of $\mathrm{Zn}$ based nanoparticles. The need for further research of the growth process and optimization of the methods is clearly indicated.

\section{ACKNOWLEDGEMENTS}

This work was supported by ERDF "Multidisciplinary research to increase application potential of nanomaterials in agricultural practice" (No. CZ.02.1.01/0.0/0.0/16_025/0007314) and by the Ministry of Education, Youth and Sports of the Czech Republic under the CEITEC 2020 project (LQ1601).

\section{REFERENCES}

[1] BOGUTSKA, K., SKLYAROV, Y.P., PRYLUTSKYY, Y.I. Zinc and zinc nanoparticles: biological role and application in biomedicine. Ukrainica bioorganica acta. 2013, vol. 1, pp. 9-16.

[2] RACCA, L., CANTA, M., DUMONTEL, B., et al. Zinc oxide nanostructures in biomedicine. Smart nanoparticles for biomedicine. 2018, pp. 171-187.

[3] SABIR, S., ARSHAD, M., CHAUDHARI, S.K. Zinc oxide nanoparticles for revolutionizing agriculture: synthesis and applications. The Scientific World Journal. 2014, vol. 2014.

[4] TAHERI, M., QARACHE, H.A., QARACHE, A.A., et al. The effects of zinc-oxide nanoparticles on growth parameters of corn (SC704). STEM Fellowship Journal. 2016, vol. 1, no. 2, pp. 17-20.

[5] REDDY, K.M., FERIS, K., BELL, J., et al. Selective toxicity of zinc oxide nanoparticles to prokaryotic and eukaryotic systems. Applied physics letters. 2007, vol. 90, no. 21, pp. 213902.

[6] SIRELKHATIM, A., MAHMUD, S., SEENI, A., et al. Review on Zinc Oxide Nanoparticles: Antibacterial Activity and Toxicity Mechanism. Nanomicro Lett. 2015, vol. 7, no. 3, pp. 219-242.

[7] ZHANG, L., JIANG, Y., DING, Y., et al. Investigation into the antibacterial behaviour of suspensions of ZnO nanoparticles (ZnO nanofluids). Journal of Nanoparticle Research. 2006, vol. 9, no. 3, pp. 479-489.

[8] CHEN, H., KOU, X., YANG, Z., et al. Shape-and size-dependent refractive index sensitivity of gold nanoparticles. Langmuir. 2008, vol. 24, no. 10, pp. 5233-5237.

[9] XIA, T., KOVOCHICH, M., LIONG, M., et al. Comparison of the mechanism of toxicity of zinc oxide and cerium oxide nanoparticles based on dissolution and oxidative stress properties. ACS nano. 2008, vol. 2, no. 10, pp. 2121-2134.

[10] BARUI, S., GERBALDO, R., GARINO, N., et al. Facile Chemical Synthesis of Doped ZnO Nanocrystals Exploiting Oleic Acid. Nanomaterials. 2020, vol. 10, no. 6, pp. 1150.

[11] WANG, Q., GENG, B., WANG, S. ZnO/Au hybrid nanoarchitectures: wet-chemical synthesis and structurally enhanced photocatalytic performance. Environmental science \& technology. 2009, vol. 43, no. 23, pp. 8968-8973.

[12] FONTE, P., REIS, S., SARMENTO, B. Facts and evidences on the lyophilization of polymeric nanoparticles for drug delivery. Journal of controlled release. 2016, vol. 225, pp. 75-86.

[13] MOEZZI, A., CORTIE, M., MCDONAGH, A. Aqueous pathways for the formation of zinc oxide nanoparticles. Dalton Trans. 2011, vol. 40, no. 18, pp. 4871-4878.

[14] CHUNG, Y.T., BA-ABBAD, M.M., MOHAMMAD, A.W., et al. Synthesis of minimal-size ZnO nanoparticles through sol-gel method: Taguchi design optimisation. Materials \& Design. 2015, vol. 87, pp. 780-787.

[15] ABRAHAMS, S., BERNSTEIN, J. Remeasurement of the structure of hexagonal ZnO. Acta Crystallographica Section B: Structural Crystallography and Crystal Chemistry. 1969, vol. 25, no. 7, pp. 1233-1236.

[16] XING, Y.J., XI, Z.H., ZHANG, X.D., et al. Nanotubular structures of zinc oxide. Solid State Communications. 2004, vol. 129 , no. 10 , pp. 671-675.

[17] CAO, H.L., QIAN, X.F., GONG, Q., et al. Shape- and size-controlled synthesis of nanometre ZnO from a simple solution route at room temperature. Nanotechnology. 2006, vol. 17, no. 15, pp. 3632-3636. 